\title{
Creative synthesis of the technical solutions in the field of industrial processes
}

\author{
Laurențiu Slătineanu ${ }^{1}$, Adelina Hrițuc $^{1, *}$, Gheorghe Nagît ${ }^{1}$, Oana Dodun ${ }^{1}$, Irina Beșliu- \\ Băncescu ${ }^{2}$, Bogdan Oroian ${ }^{1}$, Ionuț Condrea ${ }^{1}$, Carmen Botezatu ${ }^{1}$, and Mihaela Ețcu ${ }^{1}$ \\ 1"Gheorghe Asachi" Technical University of Iaşi, Department of Machine Manufacturing \\ Technology, Blvd. D. Mangeron, 59 A, Iași, Romania \\ 2"Ștefan cel Mare" University of Suceava, Department of Mechanics and Technology, Universității \\ Street 13, 720229 Suceava, Romania
}

\begin{abstract}
When a technical problem is approached, as a direct consequence of analyzing certain distinct existing solutions, an improved solution of the problem could be elaborated. Over the years, some methods able to help the designers were identified and developed. The purpose of this paper was to take into consideration and to develop a succinct comparison of some creative considered methods of solving research problems in the field of industrial processes. The first group of analyzed design method was based on the initial use of the so-called ideas diagram. The results of the analysis were used to propose solutions concerning a device for investigation of the effects developed on sharp peaks by the electrical discharges. The axiomatic design was applied as a creative design method in the case of equipment for the investigation of degradation in time of the subsystems of the computers. The reverse engineering method was considered in the case of a device for supporting the tablet in a convenient position for the user. A final succinct comparison of the three ways of synthesizing an improved solution of a technical problem from the field of industrial processes highlights the essential characteristics of the approached methods.
\end{abstract}

\section{Introduction}

The concept of "technique" comes from the Greek language and one of its meanings refers to the assembly of production tools and knowledge applied to produce goods. In the field of manufacturing engineering, there are many technical problems for which technical solutions must be found. A technical solution must be an adequate answer to a technical problem.

A general conventional classification of the technical problems shows that there are: 1 . Strategic problems, whose solutions could involve high material values and/or significant people groups; when such a problem is approached, it is important to find many solutions and subsequently to select the most convenient one, eventually by consulting other specialists; 2. Tactic problems, specific to the current situations, when a solution must be

\footnotetext{
* Corresponding author: hrituc.adelina3295@yahoo.com
} 
found in short time, and whose result does not involve high material values and/or large people groups.

The above-mentioned considerations are valid inclusively in the case of the engineer specialized in the field of manufacturing engineering when solutions of constructive or technological problems must be identified.

In the two situations (strategic problems and tactical problems), creative or routine solutions could be identified and applied. When the time for solving the approached problem is short or the solution must be found in the shortest time, usually the routine solutions could be preferred. When there is a higher duration in which a solution must be identified, the designer could try to propose a creative solution.

To efficiently search and identify creative solutions, over the times the specialists invested their efforts in defining and promoting methods able to use in a large extent the technical creativity. In this way, simpler or more complex methods for creative solving of the technical problems were proposed. Essentially, such creative methods for solving technical problems could be methods based essentially on the intuition and logical intuitive methods, respectively. As intuitive creative methods, one could consider the classical brainstorming, the lateral thinking method, lotus flower method, analogy method, advocate's method etc. Among the logical intuitive methods, the following methods could be considered: ideas diagram method, TRIZ method, axiomatic design, the method of the generalized object of the technical creation etc.

When considering the way in which a creative solution could be found, the researchers have distinct opinions. Thus, Harvey appreciated that the groups have significant creative capacities by which they could generate radical creative solutions and it is necessary to combine the groups cognitive, social and environmental resources so that finally success solutions could be identified [1].

Hoeltzel and Cheng analyzed the possibility of using knowledge-based approaches to synthesize mechanisms in a creative way. They concluded that in comparison with other design strategies, the knowledge-based computer-aided design tools could ensure higher efficiency in generation of solutions able to meet the initially assumed functional requirements [2].

The research presented in this paper aimed to take into consideration some design methods considered to be helpful in identifying and developing improved solutions for industrial processes or equipment.

\section{Methods based on the initial use of the ideas diagram}

One of the methods able to facilitate the synthesis of creative solutions for technical problems is the so-called ideas diagram methods. This method uses a graphical representation that along horizontal line includes the subassemblies identified for a possible solution of the approached technical problem. Subsequently, along vertical lines, distinct versions for each subassembly are mentioned. Both at the end of the horizontal lines and of the vertical lines, interrogation signs are also included, to highlight the idea that other subassemblies or versions of a certain subassembly could be subsequently identified. One considers that in comparison with other creative methods, the ideas diagram method suggests an open character of searching the problem various solutions, just by using these interrogation signs [3].

As one could notice, this ideas diagram method is practically a possible first stage in searching new or improved solutions for the approached problems; the applying of other methods could ensure proper conditions for identification of the wanted creative solutions. To illustrate some aspects corresponding to the possible generation of creative solutions by the initial use of the ideas diagram method, one could consider the simplified diagram 


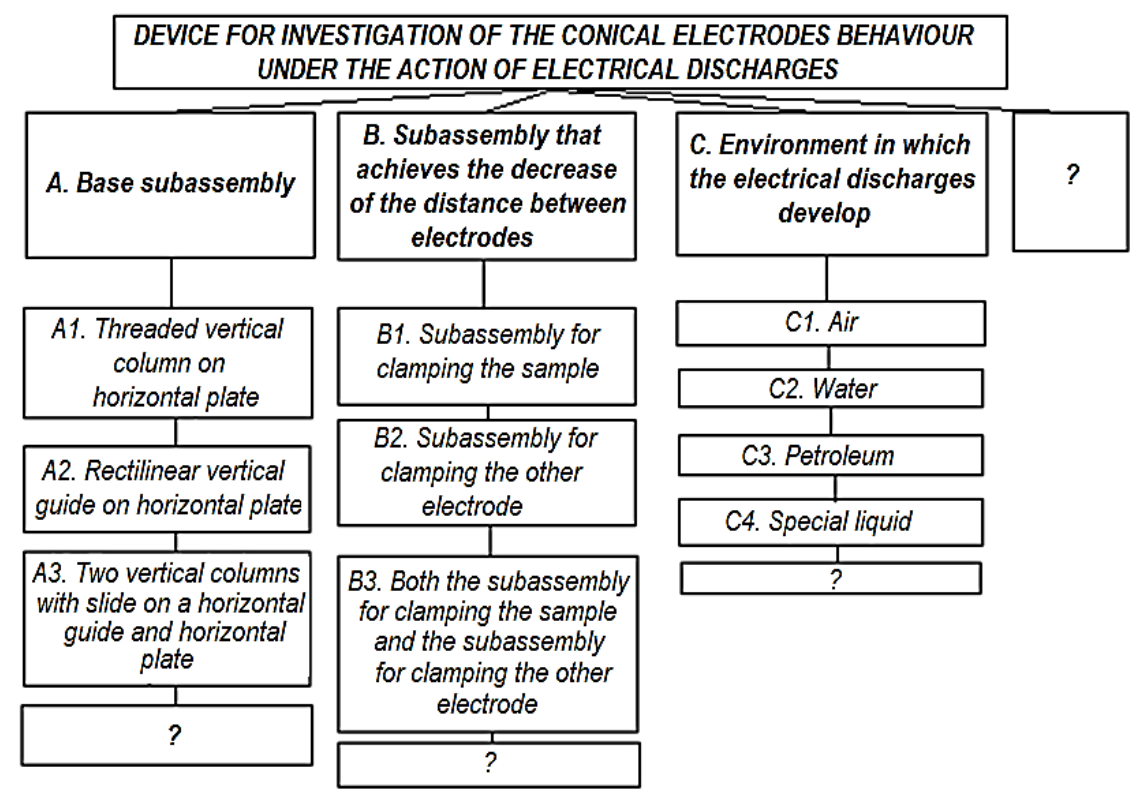

Fig. 1. Example of simplified ideas diagram valid in the case of a device for investigating the sharp peaks behavior under the action of the electrical discharges.

presented in figure 1. This ideas diagram was elaborated in the case of equipment for studying the effects of the electrical discharges on the sharp conical peaks that belong to test samples made of various electroconductive materials [4]. In the stages found after the elaboration of the graphical representation that corresponds to the ideas diagram, all the possible solutions could be highlighted, by means of the morphological method or the lexical graphical method [5-6]. In the case of the problem approached in this paper, one preferred to elaborate a morphological table in which the versions of each subassembly are symbolized by indexes added to the distinct subassemblies versions $A, B$, and $C$. In table 1 all these versions were thus mentioned. A solution $A 2 B 1 C 3$ includes practical the version 2 for the subassembly $A$, version 1 for the subassembly $B$ and version 3 for the subassembly $C$.

One could notice that the higher the number of the subassemblies and the number of the subassemblies versions are, the higher the number of the possible solutions is. In the case of the approached problem, for the three versions of the subassembly $A$ and $B$ and for the four versions of the subassembly $C$, the total number $N_{t}$ of the possible solutions will be:

$$
N_{t}=3 \cdot 3 \cdot 4=36 \text { solutions. }
$$

To analyze in detail 36 solutions, a large time could be necessary and this fact could be less convenient. To diminish the number of the solutions that could be analyzed in detail, some distinct methods could be used (global evaluation, the successive sequential method, the method of dividing in morphologies, the method of simple randomization, the method of weighted randomization etc. Taking into consideration the method of global evaluation, one considered that the solutions $A 2 B 1 C 1, A 3 B 3 C 2$, and $A 1 B 1 \mathrm{C} 2$ were considered as presenting innovative aspects and they were selected to be analyzed in detail. To develop such in detail analysis, other various methods could be applied (the value analysis method, the method of the utilities, AHP method etc.). 
Table 1. Morphological table that includes all the combinations of the versions for the subassemblies $A, B$, and $C$.

\begin{tabular}{|c|c|c|c|c|c|}
\hline \multirow{2}{*}{$\begin{array}{c}\text { Versions } \\
\text { of the } \\
\text { subassembly } \boldsymbol{A}\end{array}$} & \multirow{2}{*}{$\begin{array}{c}\text { Versions } \\
\text { of the } \\
\text { subassembly } \boldsymbol{B}\end{array}$} & \multicolumn{4}{|c|}{ Version of the subassembly $\boldsymbol{C}$} \\
\cline { 3 - 6 } & & $\boldsymbol{C 1}$ & \multicolumn{1}{c|}{$\begin{array}{c}\text { Solutions obtained by the combinations of the distinct } \\
\text { versions of the subassemblies } \boldsymbol{A}, \boldsymbol{B}, \text { and } \boldsymbol{C}\end{array}$} \\
\hline \multirow{3}{*}{$A 1$} & $B 1$ & $A 1 B 1 C 1$ & $A 1 B 1 C 2$ & $A 1 B 1 C 3$ & $A 1 B 1 C 4$ \\
\cline { 2 - 6 } & $B 2$ & $A 1 B 2 C 1$ & $A 1 B 2 C 2$ & $A 1 B 2 C 3$ & $A 1 B 2 C 4$ \\
\cline { 2 - 6 } & $B 3$ & $A 1 B 3 C 1$ & $A 1 B 3 C 2$ & $A 1 B 3 C 3$ & $A 1 B 3 C 4$ \\
\hline \multirow{4}{*}{$A 2$} & $B 1$ & $A 2 B 1 C 1$ & $A 2 B 1 C 2$ & $A 2 B 1 C 3$ & $A 2 B 1 C 4$ \\
\cline { 2 - 6 } & $B 2$ & $A 2 B 2 C 1$ & $A 2 B 2 C 2$ & $A 2 B 2 C 3$ & $A 2 B 2 C 4$ \\
\cline { 2 - 6 } & $B 3$ & $A 2 B 3 C 1$ & $A 2 B 3 C 2$ & $A 2 B 3 C 3$ & $A 2 B 3 C 4$ \\
\hline \multirow{3}{*}{$A 3$} & $B 1$ & $A 3 B 1 C 1$ & $A 3 B 1 C 2$ & $A 3 B 1 C 3$ & $A 3 B 1 C 4$ \\
\cline { 2 - 6 } & $B 2$ & $A 3 B 2 C 1$ & $A 3 B 2 C 2$ & $A 3 B 2 C 3$ & $A 3 B 2 C 4$ \\
\cline { 2 - 6 } & $B 3$ & $A 3 B 3 C 1$ & $A 3 B 3 C 2$ & $A 3 B 3 C 3$ & $A 3 B 3 C 4$ \\
\hline
\end{tabular}

To continue the creative synthesis in the case of the equipment for studying the behavior of sharp conical peaks under the action of the electrical discharge, the applying of the method of the matrix with double entries was preferred. With this aim in view, table 2 was elaborated. In this table, both along the first line and first column, the three previously selected possible solutions were mentioned.

Subsequently, each possible solution will be compared with the other two solutions versions and the score 1-0 will be established when the first solution is considered as better, $0-1$ when the second solution is appreciated as more convenient and $0.5-0.5$ when the two
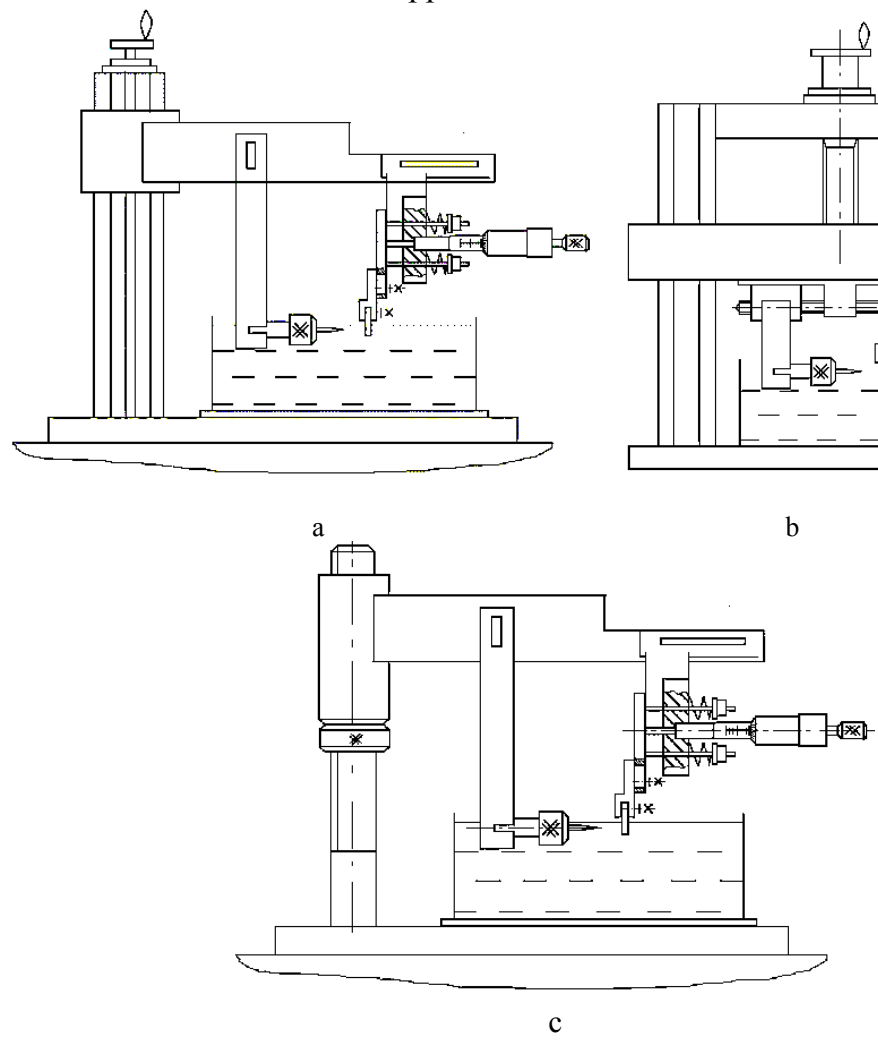

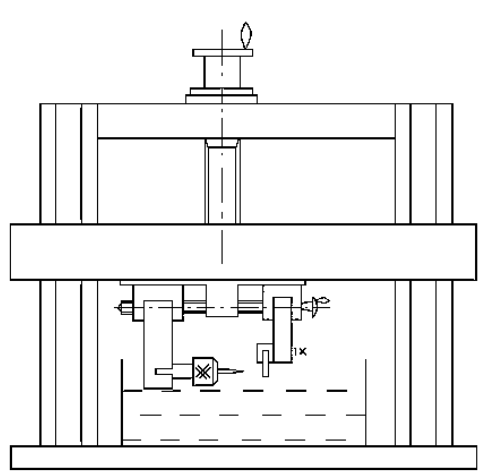

b

and $A 1 B 1 C 2$ (c). 
Table 2. Morphological table that includes all the combinations of the versions for the subassemblies $\mathrm{A}, \mathrm{B}$, and D.

\begin{tabular}{|c|c|c|c|}
\hline Possible solutions & $\boldsymbol{A 2 B 1 C 1}$ & $\boldsymbol{A 3 B 3 C 2}$ & $\boldsymbol{A 1 B 1 C 2}$ \\
\hline Column no. 1 & 2 & 3 & 4 \\
\hline$A 2 B 1 C 1$ & $\mathrm{X}$ & 0.5 & 0.5 \\
\hline$A 3 B 3 C 2$ & 0.5 & $\mathrm{X}$ & 1.0 \\
\hline$A 1 B 1 C 2$ & 0.5 & 0 & $\mathrm{X}$ \\
\hline Some of the evaluation marks & 1.0 & 0.5 & 1.5 \\
\hline Importance coefficient & 0.33 & 0.16 & 0.5 \\
\hline Order of possible solutions by considering the importance coefficient: & $A 1 B 1 C 2-A 2 B 1 C 1-$ \\
\hline
\end{tabular}

compared solutions are considered as of equal importance. The above-mentioned ratings $(0$, 1 and 0.5 ) were included in the columns 2-4 from table 2, as results of the comparisons. Simplified graphical representations of the three solutions could be seen in figure 2. In accordance with the information included in table 2 , the most convenient solution is the solution $A 2 B 1 C 1$.

When applying the more laborious method of imposed decision, initial evaluation criteria are established and subsequently each version is compared with the other version by means of each of the considered criteria $[3,5]$.

\section{Axiomatic design}

The first considerations concerning the axiomatic design were published by the professor Nam Pyo Suh in 1978. Subsequently, many other researchers have contributed to the

Table 3. Matrix that includes the functional requirements and design parameters in the case of equipment for the investigation of the accelerated degradation of the computer subsystems.

\begin{tabular}{|c|c|c|c|c|c|c|c|c|c|c|c|c|c|}
\hline Line no. 1 & \multirow{6}{*}{\multicolumn{3}{|c|}{\begin{tabular}{r|r} 
Design \\
Parameters
\end{tabular}}} & \multirow{2}{*}{\multicolumn{10}{|c|}{$\begin{array}{l}\text { Design parameter of zero order } D P 0 \\
\text { Design parameters of the first order }\end{array}$}} \\
\hline 2 & & & & & & & & & & & & & \\
\hline 3 & & & & \multicolumn{3}{|c|}{$D P 1$} & \multicolumn{3}{|c|}{$D P 2$} & \multicolumn{4}{|c|}{$\begin{array}{l}\text { e first order } \\
D P 3 \mid D P 4\end{array}$} \\
\hline 4 & & & & \multicolumn{10}{|c|}{ Design parameters of second order } \\
\hline \multirow[t]{2}{*}{5} & & & & $D P$ & $D P$ & $\overline{D P}$ & $D P$ & $D P$ & $D P$ & $D P$ & $D P$ & $D P$ & $D P$ \\
\hline & & & & 1.1 & 1.2 & 1.3 & 2.1 & 2.2 & 2.3 & 3 & 4 & 5.1 & 5.2 \\
\hline Co-lumn no.1 & 2 & 3 & 4 & 5 & 6 & 7 & 8 & 9 & 10 & 11 & 12 & & \\
\hline & & & & \multirow{2}{*}{\multicolumn{10}{|c|}{$\begin{array}{c}\text { Design parameters that correspond to each functional } \\
\text { requirement }\end{array}$}} \\
\hline 7 & $F R 0$ & $\begin{array}{c}F R s \\
\text { of the } \\
\text { first } \\
\text { order }\end{array}$ & $\begin{array}{c}\text { FRs of } \\
\text { second } \\
\text { order }\end{array}$ & & & & & & & & & & \\
\hline 8 & \multirow{3}{*}{\multicolumn{2}{|c|}{$F R 1$}} & $F R 1.1$ & $\mathrm{X}$ & $\mathrm{X}$ & & & & & & & & \\
\hline 9 & & & $F R 1.2$ & & $\mathrm{X}$ & & & & & & & & \\
\hline 10 & & & $F R 1.3$ & & $X$ & $X$ & & & & & & & \\
\hline 11 & \multirow{7}{*}{$F R 0$} & \multirow{3}{*}{$F R 2$} & FR2.1 & & & & $\mathrm{X}$ & & & & & & \\
\hline 12 & & & $F R 2.2$ & & & & & $\mathrm{X}$ & & & & & \\
\hline 13 & & & $F R 2.3$ & & & & & & $\mathrm{X}$ & & & & \\
\hline 14 & & FR3 & FR3 & & & & & & & $X$ & & & \\
\hline 15 & & $F R 4$ & $F R 4$ & & & & & & & & $\mathrm{X}$ & & \\
\hline 16 & & \multirow[t]{2}{*}{\begin{tabular}{|l|} 
FR5 \\
\end{tabular}} & FR5.1 & & & & & & & & & $\mathrm{X}$ & \\
\hline 17 & & & FR5.2 & & & & & & & & & & $\mathrm{X}$ \\
\hline
\end{tabular}


promoting and development of the theory and applications concerning the axiomatic design. Essentially, within axiomatic design, two axioms are considered. In accordance with the first axiom, the functional requirements that correspond to the problem to be solved must be independent. The second axiom shows that among many solutions for a problem, one must select the solution that needs minimum information $[7,8]$.

Certain successive stages are specific to the applying of the axiomatic design. Within a succinct example of solving a design problem by means of the axiomatic design principles, the necessity of identifying equipment for the investigation of degradation in time of the subsystems of the computers will be approached. To define the so-called customer needs, one could consider that the above-mentioned equipment is necessary to develop doctoral research. In this way, the functional requirement of zero order could be formulated: FR0: design equipment able to highlight the changes in operating conditions for certain subsystems of the computers when taking into consideration less adequate operating conditions.

If this functional requirement of zero order is deeper analyzed, the functional requirements of the second first order could be identified: $F R 1$ : ensure the change of the operating temperature; FR2: ensure the change of cooling air flow rate; FR3: ensure the deposition of a dust layer on the computer subsystem housing; FR4: ensure an adequate space where the investigated computer subsystem and various sensors could be placed; FR5: measure the computer unit performances.

To each of the functional requirement of the first order, adequate solving means must be found; these solutions are called design parameters (DPs). In the case of the equipment to be defined, such design parameters of first order could be: $D P 1$ : subsystem to controlled change of the temperature; $D P 2$ : Subsystem for controlled change of the cooling air flow rate; $D P 3$ : dust layer adhered to the investigated computer subsystem housing; $D P 4$ : recipient; DP5: computer system and software for the evaluation of the computer system performances.

Aiming at dividing the functional requirements of first order, the following functional requirements of second order could be formulated: $F R 1.1$ : measure the temperature; $F R 1.2$ : compare the temperature with the pre-established one; $F R 1.3$ : activate the heating electric resistance; FR2.1: ensure the cooling air flow; FR2.2: measure the cooling air flow rate; FR2.3: activate the airflow shutter; FR3.1: deposit a dust layer on the computer subsystem housing; FR5.1: ensure the computer subsystem; FR5.2: ensure the software for investigation of the computer system performances.

To the above mentioned functional requirements of the second order, the following design parameters could be attached: $D P 1.1$ : Temperature sensor and controller; $D P 1.2$ : temperature controller; $D P 1.3$ : temperature controller; $D P 2.1$ : Fan; $D P 2.2$ : air flowmeter; $D P 2.3$ : shutter; DP5.1: computer; DP5.2: software for the investigation of the computer system performances.

As one can see, the identification of the functional requirements and design parameters of zero, first and second order needs a periodical successive approaching both of functional requirements domain and design parameters domain; this activity constitutes the so-called zigzagging between the two domains.

A more suggestive image concerning the functional requirements, design parameters and the correlations derived from the first axiom could be obtained by including this information in a table that represents also a decision matrix (Table 2). Taking into consideration this simplified approach of some stages that correspond to the axiomatic design principles, the solution presented in figure 3 was gradually defined. The examination of the information included in table 3 shows that there are functional requirements that are met by the same design parameters; the temperature controller is used both for measuring the temperature, comparing the real temperature with the pre-established temperature and 
activating the electrical resistance. This fact highlights that possibilities of improving the proposed designed solution.

\section{Reverse engineering}

Most authors define reverse engineering by considering a concept proposed by Chikofsky and Cross in a paper published in 1990 [9], but there is the possibility of using the concept some years before. They referred to the concept of reverse engineering as a process of analyzing a subject-system to establish its components and relations among the components and to create representations of the system in another way and using a high level of abstracting. This method is also called back engineering and is used in mechanical, electronic, chemical, software engineering. At present, the reverse engineering is considered as a scientific method of analyzing a technology, equipment etc. to understand how they were designed or function and thus identify the ways of improving and increasing the performances of the analyzed system.

To succinctly illustrate the stages of applying reverse engineering, the solution of a book holder is subsequently analyzed.

1. Identification of the product to be analysed. As above-mentioned, the case of a book holder will be taken into consideration. As an initial solution, the main image presented in the U.S.A. patent no. 5,351,927 [10] will be used (fig. 4).

2. Investigation or disassembling of the information concerning the structure and the way of using the original system. One could notice here that the original system includes a proper book holder placed at the end of a vertical telescopic tubular column. This column is attached to a base component that includes 3 rollers designed to provide a displacement of the book holder assembly in a horizontal plane. Some cylindrical joints facilitate the angular positioning of the proper book holder.

3. Use of the accumulated information to generate a modified version of the original

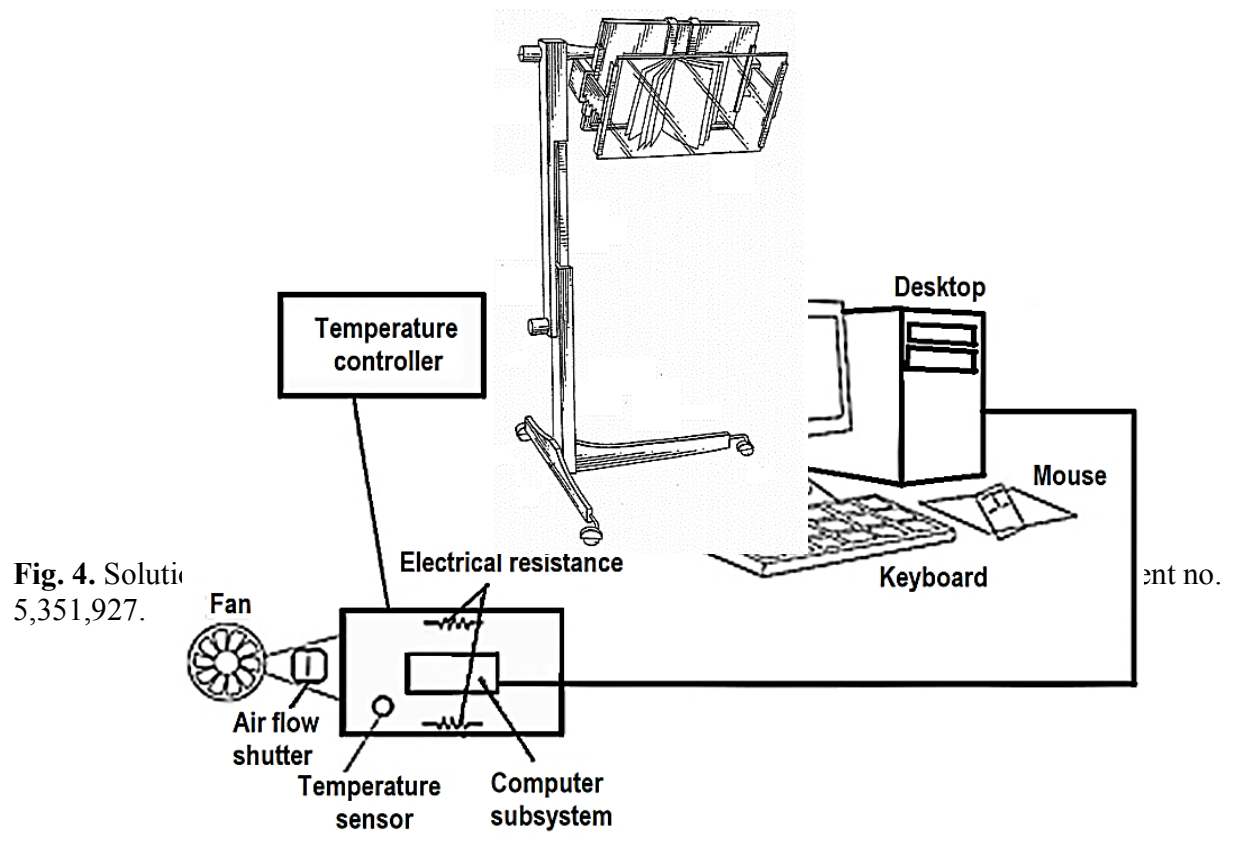

Fig. 3. Solution for equipment aiming to test the computer system components. 
system. Taking into consideration the information achieved by the investigation of the solution included in the U.S.A. patent no. 5,351,927, the solution presented in figure 5 was gradually developed [11]. As someone could see, the proposed version of the book holder uses a flange piece by which it is fixed to the wall and to which book support is attached by means of a telescopic joint and a spherical joint, respectively. To attach the book to the proper book support, a cover of transparent material is used. The position of the cover could be changed for distinct books thicknesses. To ensure the adequate illumination of the book, a led type lamp could be clamped at the end of the flexible tube mounted on the proper book support. As the main improvement of this solution in comparison with the initially analyzed book holder, one could take into consideration the possibility of attaching the book holder to a wall and the inclusion of a light source.

4. Practical generation of a new product could be appreciated as one of the final stages of applying the reverse engineering method.

As one can see, the proposed solution does not copy the components of the initial system and, in this way, the copyright laws are not violated.

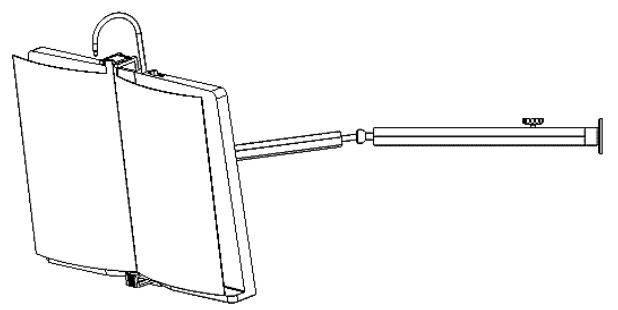

Fig. 5. Solution for a book holder that could be developed by applying the principles of reverse engineering [11].

The main advantages of the reverse engineering method derive from the diminishing of the time for documentation and for generation of the wanted solution; practically, the attentive examination of the wanted object existing version could directly suggest certain possibilities of improving the initial solution to a creative designer. As a disadvantage, one could take into consideration the possible psychological obstacle represented by the existing solution, which can prevent the designer from seeing how other solutions, quite different from the original solution, could be designed.

\section{Succinct comparative analysis}

Some succinct details concerning the use of certain creative methods in solving the problems of synthesizing improved equipment were previously presented. There are also other creative methods susceptible to be applied in synthesizing technical solutions intended to be used within various processes. Each researcher or designer could select the method he considers the adequate for solving his technical problem. To develop a short comparison of the above-mentioned creative methods, some evaluation criteria were identified. Thus, one took into consideration the method complexity level (one considers that a method involving many stages is more complex and time-consuming), the necessity to consider an initial possible known (existing) solution, the presence of stages when a retaking of the synthesizing process is necessary. In this evaluation, three qualitative criteria (high, medium and low) were used; one could accept that in establishing these qualitative criteria some subjective consideration could be present (Table 4). In fact, as above mentioned, over the times, each researcher or designer could establish his own way of efficient creative developing of new or improved equipment or processes, but it is important to have information concerning the existing creative methods that could be used 
to solve the technical problems or just to combine the characteristics or stages of distinct methods to more efficiently solve the assumed problems.

Table 4. Succinct evaluation of the three creative methods applied in synthesizing improved equipment or processes.

\begin{tabular}{|c|c|c|c|c|}
\hline $\begin{array}{c}\text { Method } \\
\text { no. }\end{array}$ & $\begin{array}{c}\text { Method or group methods } \\
\text { name }\end{array}$ & $\begin{array}{c}\text { Complexity } \\
\text { level }\end{array}$ & $\begin{array}{c}\text { Need for an } \\
\text { initial } \\
\text { solution }\end{array}$ & $\begin{array}{c}\text { Necessity of re- } \\
\text { taking certain } \\
\text { stages }\end{array}$ \\
\hline 1 & $\begin{array}{c}\text { Methods based on the use } \\
\text { of the ideas diagram }\end{array}$ & High & High & Medium \\
\hline 2 & Axiomatic design & Medium & Low & Low \\
\hline 3 & Reverse engineering & Medium & High & Medium \\
\hline
\end{tabular}

\section{Conclusions}

The problem of using design methods able to usually lead to new or improved solutions of equipment or processes has captured the interest of the researchers from the field of design activities. Over the years, many methods of creative solving of the technical problems were proposed and promoted. Three of these methods were taken into consideration in the research presented in this paper to be applied in finding a solution for a certain problem. Thus, some methods based essentially on the use of ideas diagram were used to identify a device for studying the behavior of conical peaks under the action of the electrical discharges, while some principles of the axiomatic design were applied to develop equipment for the investigation of the computer subsystems in less convenient operating conditions. In a third example, the reverse engineering method was applied to find a solution for a book holder. A succinct comparison of these creative methods of solving technical problems was achieved by considering some qualitative criteria. One concluded that the use of one of the presented creative methods or even of other creative methods depends on the researcher abilities, knowledge and confidence in the possible success of using a certain creative method or a group of creative methods. Another conclusion takes into consideration the necessity that, when the designer has a certain professional experience, he could elaborate a first solution without a deep investigation of the available literature, so that the designer could not be influenced by the existing solutions. Only in a second stage, a comparison of his solution with the solutions found in the available literature could contribute to the improving of his initial version of solution.

\section{References}

1. S. Harvey, Acad. Manage. Rev. 39, 324-343 (2012)

2. D. A. Hoeltzel, W.-H. Chieng, Comput. Aided Design 22, 57-67 (1990)

3. V. Belous, Inventics (Asachi Publishing House, Iași, 1992)

4. M. Ețcu, L. Slătineanu, S.-N. Croitoriu, 22nd International Conference on Innovative Manufacturing Engineering and Energy - IManE\&E 2018, MATEC Web Conf., 178, 03001 (2018)

5. L. Slătineanu, P. Dușa, Management of technological innovation (Tehnopress Publishing House, Iaşi, 2002)

6. G. Nagîț, Techniques and methods for creativity stimulating (Tehnica-Info Publishing House, Chişinău, 2001)

7. N.P. Suh, Axiomatic Design: Advances and Applications (Oxford University Press, New York, 2001)

8. L. Slătineanu, Industrial property (Performantica Publishing House, Iași, 2015)

9. E.J. Chikofsky, J.H. Cross, IEEE Software 7(1), 13-17 (1990) 
10. R.J. Howell, U.S. Patent no. 5,351,927 (1994)

11. A. Hrițuc, Romania patent application no. A/ 01093/ 2018 (2018) 